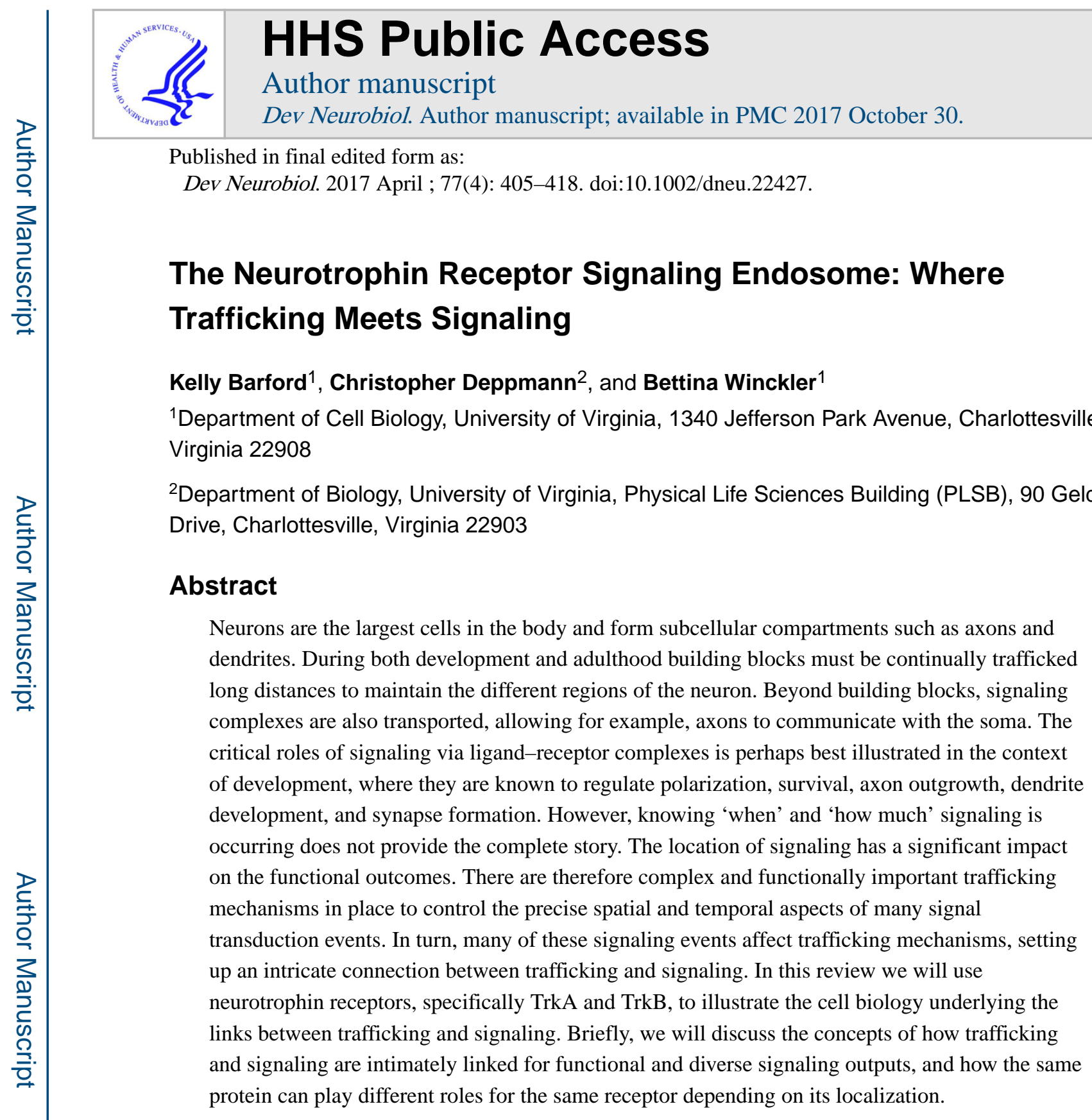

\title{
Keywords
}

endocytosis; Rab proteins; transcytosis; Coronin1a; Trk receptors

\section{INTRODUCTION: MEET THE PLAYERS}

\section{The Neurotrophins and Their Receptors}

The Trk family of receptor tyrosine kinases (RTKs) include TrkA, TrkB, and TrkC which bind with high affinity to NGF, BDNF/NT-4, and NT-3 respectively. Collectively, this class of structurally related ligands is called the neurotrophins. Like many other RTKs, Trk receptors initiate classical signaling including the PLC $\gamma, \mathrm{PI} 3 \mathrm{~K}$, and Ras/MAPK pathways

Correspondence to: B. Winckler (bwinckler@virginia.edu). 
(Patapoutian and Reichardt, 2001). Activation of these pathways initiates a large number of downstream events including local signaling to the cytoskeleton resulting in axon branching as well as transcriptional changes. All neurotrophins can also bind to and activate p75-

NGFR, which can either cooperate with or antagonize Trk function depending on cellular context. There are many excellent reviews on p75-NGFR, and its function will not be addressed in detail here (Barker, 1998; Meeker and Williams, 2015).

Neurotrophin signaling has been widely studied in the sympathetic and sensory nervous systems. During development, sympathetic neurons extend axons, which express TrkA, into the periphery along the vasculature and encounter NGF upon reaching their final target (DiStefano et al., 1992; Grimes et al., 1996; Saragovi et al., 1998; Beattie et al., 2000; Howe et al., 2001; Shao et al., 2002; Howe and Mobley, 2004; Valdez et al., 2005). At the final target, neurons encounter limiting amounts of NGF which is only sufficient to support the survival of $\sim 50 \%$ of neurons. Neurons that do not receive sufficient NGF die via apoptosis (Detwiler, 1924; Hamburger, 1934; Bueker, 1948; Levi-Montalcini and Hamburger, 1951). If either TrkA or NGF are knocked out in mice, sympathetic and nociceptive neurons are lost, indicating the necessity for both TrkA and NGF in survival signaling (Crowley et al., 1994; Smeyne et al., 1994). The most striking observation for NGF signaling in both sensory and sympathetic neurons is that NGF is only secreted from targets in the periphery during development, yet it elicits responses in the cell bodies residing many millimeters away in the ganglia. It thus has been a long-standing question in the field: how is a ligand binding its receptor at the distal axon tip able to elicit transcriptional changes in the soma?

\section{The Signaling Endosome}

Evidence is now overwhelming that NGF-dependent signaling is propagated from the distal axon to the soma through physical translocation of the NGF-TrkA complex in an endosome. Upon encountering NGF in the distal axon, TrkA binds to NGF and the complex is internalized into endosomes. This endosome is then transported retrogradely by dynein to the cell body (Heerssen et al., 2004) where it elicits survival signaling (Riccio et al., 1997, 1999). These "signaling endosomes" (SEs) contain activated TrkA and are associated with adaptors and effector proteins that mediate downstream signaling cascades (Grimes et al., 1996; Watson et al., 2001; Delcroix et al., 2003; Harrington et al., 2011).

A major focus of the neurotrophin field has been survival signaling, but it is now appreciated that TrkA has many other functional roles including differentiation, axon growth/branching, acquisition of neurotransmitter phenotypes, and changes in metabolism (Snider, 1994; Deshmukh et al., 1996; Glebova and Ginty, 2005; Sharma et al., 2010; Harrington et al., 2011; Suo et al., 2015). Two major open questions are whether these diverse functions are controlled by the same signaling endosomes that mediate survival signaling and how bifurcation of signaling outputs is achieved with such specificity. Novel trafficking events that precede or follow NGF-TrkA binding in the distal axon have been discovered which are critical for both survival signaling in the soma and local signaling in axon terminals (Shao et al., 2002; Ascaño et al., 2009; Mitchell et al., 2012; Suo et al., 2014). 
TrkA Trafficking Pathways

TrkA trafficking can be subdivided into several distinct events (Fig. 1). First, TrkA can undergo constitutive endocytosis and recycling in the soma in the absence of ligand. Upon NGF signaling, these endosomes are transported anterogradely to the axon. (Ascaño et al., 2009). Second, in the axon, TrkA encounters NGF and endocytoses into a retrogradelytransported signaling endosome (Howe et al., 2001; Ye et al., 2003). Lastly, the signaling endosome can be exocytosed on the soma membrane and subsequently re-internalized (Suo et al., 2014). The first endocytic event, constitutive endocytosis and subsequent transcytosis, is responsible for anterograde transport of TrkA to the axon in a positive-feedback loop initiated by NGF signaling. The second endocytic event, internalization in the distal axon after NGF binding, forms the signaling endosome. It has long been appreciated that TrkA signaling differs before and after internalization (Zhang et al., 2000). Formation of the signaling endosome therefore is crucial for adequate TrkA signaling. The third endocytic event, here termed "signaling transcytosis," has only been recognized recently (Suo et al., 2014), is still incompletely understood, and could be a potential mechanism for lysosomal avoidance and/or specification of signaling outputs.

It appears then that a multitude of TrkA trafficking events contribute to NGF-TrkA function. A major open question is whether and how endosome formation, trafficking, maturation and identity affect the specific signaling outcomes for TrkA, and whether there are functionally distinct sets of signaling endosomes that differ in terms of location, signaling, and associated effectors. For instance, it is not known whether after undergoing signaling transcytosis, the re-internalized Trk receptor is still signaling, and what role this trafficking route plays in TrkA signaling. In addition, it is largely still unknown what regulators allow TrkA to undergo multiple internalization events with differential outcomes. Since axon extension, survival, and synapse formation are all NGF-dependent in sympathetic neurons, a major challenge in the field is to determine whether particular trafficking or signaling events underlie a particular developmental outcome.

\section{The Different "Flavors" of Endosomes}

The Trk-signaling complex must move correctly through the endosomal pathway in order to elicit functional outputs such as survival signaling, axon outgrowth, and dendrite development. The endosomal pathway is well studied in non-neuronal cells and is summarized below and diagramed in Figure 2. In mammalian cells, cargos can be endocytosed by a number of distinct pathways, which largely converge in a sorting endosome, also called the early endosome. In the early endosome, cargos have several choices. They either sort back to the membrane locally (i.e. local recycling), or to a distant final membrane destination (i.e. long-distance recycling), traffic towards lysosomes for degradation, or traffic back to the trans-Golgi network. The early endosome is thus a compartment that determines a cargo's final fate (Jovic et al., 2010).

Along a receptor's trafficking route, it associates with a variety of different membraneassociated proteins, which direct the cargo down its destined path. Many classes of proteins have been implicated in regulating sorting decisions in the endosome, including small GTPases (such as Rab and Arf proteins), SNARE proteins, sorting nexins, tethering factors, 
cytoskeleton, motor proteins, and others (Grosshans et al., 2006; Naslavsky and Caplan, 2011; Bonifacino, 2014; Wandinger-Ness and Zerial, 2014; Burd and Cullen, 2016).

Some of the best-understood endosomal trafficking regulators are the Rab family of small GTPases, and they are widely considered to be the master regulators of membrane trafficking. Activated Rabs play a critical role in vesicle fusion, transport, and maturation via recruitment of specific effectors, and localize to specific subsets of endomembrane compartments. They are thus frequently used as markers to identify different subsets of endosomes (Grosshans et al., 2006) (Fig. 2). For instance, the early/sorting endosome is marked by Rab5. Rab5-positive early endosomes frequently contain either APPL1 or EEA1 (early endosome antigen 1) (Wandinger-ness and Zerial, 2014). APPL1 in particular has been implicated in TrkA signaling (Lin et al., 2006). The early endosome can subsequently sort cargo into the recycling pathway or the late endosome/lysosome pathway (Jovic et al., 2010). Rapid, local recycling to the same area on the membrane occurs via Rab4 from the early endosome in Rab4-positive tubular carriers (Wandinger-Ness and Zerial, 2014). Alternatively, Rab11 marks endosomes that recycle more slowly and often to different areas of the cell. In fibroblasts, the Rab11-positive recycling compartment is also referred to as the ERC (endosomal recycling compartment) and is localized stably near the centrosome (Horgan et al., 2010). Cargos can recycle from this more stable ERC compartment using Rab11 with slower kinetics (Sheff et al., 1999).

The late endosome is marked by Rab7 and constitutes the intermediate compartment between the early endosome and the lysosome (Huotari and Helenius, 2011). Lysosomes also carry Rab7 as well as other characteristic proteins such as LAMPs (lysosome-associated membrane proteins) and lysosomal hydrolases (such as cathepsins), which are responsible for degradation (Klumperman and Raposo, 2014; Scott et al., 2014). While the early endosome structurally resembles a network of tubules with various vacuolar domains, late endosomes and lysosomes have a very different morphology. Late endosomes are morphologically characterized by having intralumenal vesicles (ILVs) surrounded by a limiting membrane, forming what is called a multivesicular body (MVB) (Klumperman and Raposo, 2014). ILVs form through portions of the limiting membrane budding into the lumenal space, a process requiring the ESCRT complex proteins (Hanson and Cashikar, 2012; Henne et al., 2013). Late endosomes ultimately fuse with lysosomes, which are filled with electron-dense material and membranes. Cargos delivered to the lysosome are degraded by acidic hydrolases active at the low acidic $\mathrm{pH}$ of lysosomes. MVBs are, therefore, typically thought of as intermediate endolysosomal compartments on their progression to the lysosome (Woodman and Futter, 2008; Klumperman and Raposo, 2014). While late endosomes are marked by the presence of Rab7 on the limiting membrane, some lysosomes lose Rab7 association but retain other markers such as LAMPs on their limiting membranes. It should be noted that while the canonical role of lysosomes is degradation, there is clear evidence that in many cell types, lysosomes can themselves be secretory. Here, lysosomes are able to fuse with the plasma membrane and secrete their ILVs as exosomes. Rab3 and Rab27a have been shown to play a critical role in endolysosomal secretion including exosomes (Blott and Griffiths, 2002; Chiang et al., 2011; Lettau et al., 2015). While this has mainly been studied in hematopoetic cell types and cancer, it is likely that this can occur in other cells, including neurons (Arantes and Andrews, 2006). 
There are over 50 Rabs in the mammalian genomes and little is known about most of them in any cell type. The best studied endosomal Rabs (Rab4, 5, 7, 8, 11) have mostly been investigated in fibroblasts. In neurons, somatic endosomes resemble the endosomes in fibroblasts in many respects. Even though endosomes in axons and in distal dendrites are presumed to be similar to the somatic ones, there are some clear differences between axonal and somatodendritic endosomes. Little is known about the functional consequences of these differences. One of the known differences is with respect to early endosomes. In the soma and dendrites, Rab5 and EEA1 are present. In the axon, on the other hand, EEA1 is not present, but Rab5 is still associated with early endosomes (Wilson et al., 2000). Some neuronal-specific proteins (such as the small transmembrane protein NEEP21) are also excluded from axonal endosomes and only found in somatodendritic endosomes (Yap et al., 2008; Lasiecka et al., 2014).

\section{Effectors and Regulators of Rab Proteins - Why the Rab "Flavor" Matters}

How is the identity of the endosome functionally relevant? There are many different Rabs, and each one has their own set of effector proteins that can associate with it at a given time (Wandinger-ness and Zerial, 2014). Rab proteins are small GTPases that are active when GTP-bound. In their active GTP-bound state, they recruit a variety of effectors to the endosome (Bucci et al., 2014; Hutagalung and Novick, 2011) which then can affect the behavior of this endosome. Essentially, each Rab protein can give the same signaling endosome a different set of effector proteins, thus providing a direct way of diversifying the pool of signaling endosomes.

Rab effectors fall into several classes: effectors that recruit proteins that promote tethering and fusion with other compartments, recruit motor proteins that can move the compartment to a different location in the cell, recruit proteins that regulate cargo inclusion and coat protein association for vesicle formation, and recruit proteins that regulate actin cytoskeleton. Therefore, the specific Rab that is present on a specific endosome decides whether or not to include a particular cargo into the carrier, to bud off a carrier, to fuse with another compartment, to recycle or degrade, and to move to another place in the cell. Just to name a few examples: (1) Both Rab5 and Rab7 can link to the retrograde dynein motors via one set of adaptors, or link to kinesin motors via a different set of adaptors (Zhen and Stenmark, 2015); (2) Rab11 can link to kinesin, but also to the actin motor myosin $\mathrm{Vb}$ (Schafer et al., 2014); (3) Rab5 recruits tethering factors, such as EEA1 (Simonsen et al., 1998; Callaghan et al., 1999); (4) Rab7 recruits HOPS complex and ESCRT to promote maturation of intralumenal vesicles (Wang and Hong, 2006; Lin et al., 2013). TrkA has been shown to traffic in Rab5-, Rab11-, or Rab7-endosomes, and TrkA-containing endosomes are thus likely to have different association with SNARE fusion machinery, kinesin or dynein motors, or association with actin filaments (Delcroix et al., 2003; Zhang et al., 2013; Suo et al., 2014) depending on the associated Rab. An endosome's association with SNARE fusion machinery, motors, and the cytoskeleton can affect its trafficking fate (Wojnacki and Galli, 2016). Signaling downstream of TrkA can thus be regulated in terms of duration and location by means of either avoiding (or not) fusion with lysosomes, moving retrogradely in axons, moving into dendrites, or fusing with other compartments or the plasma membrane. Whereas the association and significance of regulating association with motor proteins via 
Rabs in terms of regulating neurotrophin signaling is well established, how regulating fusion with other compartments (such as lysosomes or the plasma membrane) requires Rabmediated regulation of SNARE function is not well established. What is clear, though, is that the regulation of trafficking of TrkA through different endosomal compartments affects its signaling output.

Conversely, signaling can also affect trafficking. One example of this is that Rabs are activated and inactivated by proteins which themselves are subject to regulation. When, where and for how long a particular Rab protein is active thus regulates trafficking downstream of signaling. Rabs are activated by GEF proteins (GDP exchange factor) that exchange GDP for GTP on the Rab. They are inactivated by GTPases activating proteins (GAP) that accelerate the intrinsic GTPase activity of Rabs (Barr and Lambright, 2010). There are a large number of both GEFs and GAPs, and while their regulation is currently poorly understood, they clearly constitute a rich field of exploration for the future. For example, several Rabs are regulated by phosphorylation: Rab5b is a substrate for the kinase LRRK2 (which is implicated in Parkinson's disease) (Yun et al., 2015) and Rab11 is regulated by the kinase LMTK1 in a cdk5-dependent manner (Takano et al., 2012). Kinase signaling cascades are thus upstream of activating Rab proteins.

Not surprisingly, several Rab proteins have been implicated in Trk receptor trafficking (Delcroix et al., 2003; Ascaño et al., 2009; Harrington et al., 2011; Zhang et al., 2013; Suo et al., 2014). Even though the precise association both spatially and temporally with the correct Rab protein is critical for Trk receptor function, and disruption of this association causes disruption of NGF-dependent processes, the exact time and place of how various Rabs regulate NGF-TrkA trafficking and signaling are still incompletely understood.

\section{TRKA SIGNALING ENDOSOME: TRAFFICKING REGULATES SIGNALING AND SIGNALING REGULATES TRAFFICKING}

It is now quite clear that endosomal identity and precise trafficking are crucial to neurotrophic signaling. In the following, we will take each of the three TrkA trafficking pathways outlined in Figure 1 and discuss the current evidence that trafficking regulates signaling and signaling regulates trafficking. We will start with the retrograde axonal pathway since it is by far the most extensively studied (Zweifel et al., 2005; Yamashita and Kuruvilla, 2016).

\section{Trafficking Affects Signaling: How Is Distal NGF Signaling Transmitted to the Soma?}

Neurotrophin binding causes dimerization, autophosphorylation, and internalization of Trk receptors. Phosphorylated Trk is active and initiates canonical RTK signaling including activation of the PI3K/Akt, Ras/ERK, and PLC $\gamma$ pathways. These signaling pathways have been extensively studied and will not be reviewed here (Kaplan and Stephens, 1994; Huang and Reichardt, 2003). NGF-mediated signals must travel retrogradely back to the soma to exert many of their effects during development, i.e. trafficking regulates signaling. Indeed, when NGF is added exclusively to neuronal cell bodies, the neurons survive but retract their axons (Campenot, 1977). Additionally, dynein transport back from the distal axon is needed 
to ensure cell survival (Heerssen et al., 2004). Therefore, internalization of NGF-TrkA in the distal axon and retrograde transport are both necessary features for signaling cascades that allow a neuron to survive and develop correctly.

\section{Signaling Affects Trafficking: What Is the Regulation That Biases Activated TrkA Endosomes to Move Retrogradely to the Soma?}

Correct trafficking back to the soma is integral for survival signaling and dendrite development, and much work has been done to understand how TrkA signaling endosomes travel retrogradely down the axon (Heerssen et al., 2004; Sharma et al., 2010; Zhou et al., 2012). The identity of the endosomes that carry activated Trk receptors retrogradely down the axon has been widely studied but remains controversial.

After internalization, Trk receptors are endocytosed into a signaling competent compartment. NGF-TrkA complexes have been shown to enter a Rab5-positive early endosome upon endocytosis in the distal axon (Deinhardt et al., 2006; Philippidou et al., 2011). Whether or not the signaling endosome is trafficked retrogradely in a Rab5containing compartment is unclear. In the case of TrkA, there have been reports that the signaling endosome is a Rab5-positive early endosome, as well as reports that it is a Rab7positive late endosome (Delcroix et al., 2003; Zhang et al., 2013), and both might be the case. It is thought that activated TrkA in endosomes recruits and activates RabGAP5, a GAP that inactivates Rab5 (Liu et al., 2007; Bucci et al., 2014). The authors find that inactivation of Rab5 actually prolongs signaling in PC12 cells and neurite outgrowth arguing that signal duration is controlled by the residence time in the maturing endosome. Delay in maturation prolongs signaling. The activation of RabGAP5 by activated TrkA is thus a clear example of how the cargo itself (in this case TrkA) takes charge of its own endosomal fate by regulating the activity of Rab5, i.e. signaling regulates trafficking.

On the other hand, there is evidence that Rab7 escorts the signaling endosome back to the soma (Saxena et al., 2005; Zhang et al., 2013) where it continues to signal. In apparent contradiction, interference with Rab7 function via a dominant-negative Rab7 also enhances NGF signaling, again suggesting that slowing progression of endosomes towards the late endosome increases signal strength. Again, this work was performed in PC12 cells and local and long distance signaling endosomes could not be distinguished. Further work will be needed to untangle whether conversion to Rab7 endosomes is necessary for some NGFmediated signaling events or whether conversion shortens signaling.

For TrkB, a Rab5 to Rab7 early-to-late endosome conversion event in the distal axon has been shown to result in TrkB trafficking retrogradely back to the soma in a Rab7-containing late endosome (Deinhardt et al., 2006). It is thus an open question if there is only one homogeneous retrograde signaling endosome, or if multiple distinct classes of signaling endosomes (Rab5 and Rab7-positive) reach the soma from the axon. If there were multiple molecularly distinct populations of signaling endosomes, what would be the functional consequence of such signal endosome diversity?

Dev Neurobiol. Author manuscript; available in PMC 2017 October 30. 


\section{How Trk Levels Are Maintained in the Distal Axon: Anterograde Trafficking of Trk Receptors from the Soma to the Axon}

Trk receptors are synthesized and trafficked to the plasma membrane by way of the ER and Golgi. TrkA is needed at the distal tips of axons in order to initiate all of its functional outputs, yet not all newly synthesized TrkA is trafficked directly to the axon (Ascaño et al., 2009). How is an adequate amount of Trk receptors targeted to and maintained on the axonal surface in order for proper neurotrophin signaling to take place, and is there dynamic scaling of Trk receptor levels to match physiological needs? Importantly, Trk receptor levels are dynamically regulated through feed-forward mechanisms of neurotrophin signaling and endosomal trafficking. NGF activation of TrkA increases the amount of TrkA receptor available on the axon through three mechanisms: transcytosis, local insertion from endosomes in distal axons, and transcriptional regulation (Deppmann et al., 2008; Ascaño et al., 2009).

\section{Signaling Affects Trafficking: Transcytosis of Trk Receptors to the Axon-In}

2009, Kuruvilla et al. made the unexpected discovery that naive TrkA undergoes constitutive endocytosis and local recycling in the soma without binding NGF (pathway 1 in Fig. 1). Much of the somatic TrkA thus resides in endosomes in the soma (Ascaño et al., 2009). When NGF is added to the axon, the somatically recycling pool is mobilized downstream of NGF signaling to enter the axon and traffic to the distal axon tip via endosomes. This pathway of endocytosis at the soma and subsequent transport to the axon in endosomes is dependent on NGF signaling and termed transcytosis, borrowing a term from epithelial cell biology. Transcytosis to the axon is used by several other membrane receptors, including cell adhesion molecules and integrins for localization to the axon (Ikonen et al., 1993; Yamazaki et al., 1995; Wisco, 2003; Yap et al., 2008; Ascaño et al., 2009; Eva et al., 2010).

Anterograde transcytosis of TrkA is thus a clear example of NGF signaling regulating the trafficking of TrkA.

It is unclear whether transcytosis is necessary for all anterograde trafficking or if a portion of TrkA traffics to the axon directly after synthesis. Additional regulators of Trk anterograde transport have been discovered. For example, sortilin, a sorting receptor, interacts with all 3 Trk receptors to enhance their anterograde transport (Vaegter et al., 2011). However, it has not been determined at which step in anterograde transport sortilin is involved, constitutive endocytosis, transcytosis of TrkA, or direct axonal transport from the trans-Golgi network (Vaegter et al., 2011) and whether or not signaling affects sortilin-mediated trafficking of Trk receptors.

Trafficking Affects Signaling: Anterograde Transport of Trks-After somatic endocytosis, TrkA can be transported anterogradely in Rab-11 containing recycling endosomes to the axon (Ascaño et al., 2009). This makes sense given that Rab11 is known to associate with kinesin motors (Schonteich et al., 2008; Simon and Prekeris, 2008; Esteves da Silva et al., 2015), and recycling endosomes avoid degradation while being capable of longdistance transport. Indeed, if dominant-negative Rab11 is transfected into sympathetic neurons grown in compartmentalized cultures and NGF is added only to the distal axon, axon growth is significantly impaired. Similarly, if the cultures are incubated with the 
recycling blocker monensin, TrkA signaling is greatly impaired (Ascaño et al., 2009), showing a clear example of how TrkA trafficking can affect downstream signaling. Maintaining adequate levels of TrkA on the axonal plasma membrane via regulated anterograde transport is thus critical for setting signaling and appropriate functional outputs.

Similarly, correct TrkB anterograde transport is necessary for adequate downstream signaling. Two separate mechanisms, involving Rab27B or c-Jun $\mathrm{NH}_{2}$-terminal kinase interacting protein 3 (JIP3), have been shown to mediate anterograde transport of TrkB to the axon and disruption of either effects TrkB downstream signaling. Rab27B is known to be present on secretory vesicles, thus supporting the hypothesis that it traffics newly synthesized TrkB into the axon (Chiang et al., 2011). Rab27B interacts with TrkB indirectly by binding to the TrkB effector Slp1. Slp1 also binds to CRMP-2, forming a complex with TrkB, Rab27B, and CRMP-2. CRMP-2 attaches this complex to kinesin-1 and transports it into the axon (Arimura et al., 2009). Knockdown of either Rab27B, CRMP-2, or Slp1 cause a decrease in ERK1/2 phosphorylation downstream of TrkB activation by BDNF (Arimura et al., 2009). These data imply that TrkB anterograde transport to the axon is required for adequate BDNF/TrkB signaling.

In addition to Rab27B, JIP3 also transports TrkB anterogradely in the axon. JIP3 binds to TrkB outside of its kinase domain and acts in supplement to the Rab27B dependent mechanism to deliver TrkB to the axon (Huang et al., 2011). JIP3 has been shown to be present on vesicles where it recruits kinesin-1 for transport (Bowman et al., 2000; Setou et al., 2002; Marchesin et al., 2015). Therefore, TrkB is transported to the axon in a vesicle by kinesin-1 synchronously through Rab27B and JIP3. Knockdown of JIP3 causes a decrease in anterograde transport of TrkB headed to the axon, but not to the dendrites, showing specificity of anterograde transport of TrkB. This anterograde transport is necessary for TrkB signaling, as JIP3 knockdown decreases the amount of phosphorylated ERK in the axon (Huang et al., 2011). Thus, trafficking affects the signaling of both TrkA and TrkB.

\section{What Happens When the Signaling Endosome Reaches the Soma?}

The trafficking mechanisms that the signaling endosome undergoes upon reaching the soma are not yet well known. It is known that TrkA maintains persistent signaling to induce transcriptional changes after reaching the soma. In sympathetic neurons grown in compartmentalized cultures, TrkA signaling endosomes accumulate for 6 hours before disappearing, but a pool TrkA signaling endosomes can survive for up to 25 hours in the soma without being degraded (Suo et al., 2014). Somatic signaling endosomes thus need to escape fusion with lysosomes in order to continue signaling. How the fate of somatic TrkA signaling endosomes is regulated has only begun to be addressed, but regulation of trafficking is likely a major factor.

Signaling Transcytosis: Intertwining Trafficking and Signaling-TrkA signaling endosomes must survive for a long time in the soma. Indeed, it has been shown that TrkA signaling endosomes can survive in the soma for up to 25 hours (Suo et al., 2014). Recent experiments to better understand what happens to signaling endosomes after they reach the soma have uncovered an unexpected pathway: local recycling in the soma, a process we 
refer to as "signaling transcytosis" (Fig. 1). Upon reaching the soma, the TrkA signaling endosome is exocytosed on the soma membrane and then re-internalized (Suo et al., 2014). What is the purpose of this recycling, and is it induced by a TrkA signaling cascade? One possibile function of this pathway is that signaling transcytosis allows for some signaling endosomes to switch compartment identity: a signaling endosome arriving retrogradely risks degradation, especially when associated with Rab7. Exocytosis and subsequent endocytosis would allow for re-partitioning into Rab11-positive recycling endosomes. Consistent with this hypothesis, signaling endosomes are found associated with Rab11 in the soma (Suo et al., 2014).

As mentioned above, there are data supporting the idea that retrograde SEs are of diverse "flavors", either Rab5 or Rab7. If the retrograde TrkA SE is a Rab5-positive early endosome, then it would easily be able to mature into a Rab11-positive recycling endosome. However, if retrogradely trafficked SEs are Rab7-positive late endosomes, their canonical fate would be degradation. In this case, it would be unlikely for SEs to survive for 25 hours in the soma (Suo et al., 2014). Indeed, to the best of our knowledge there is no current model that shows Rab7-positive late endosomes progressing into Rab11-positive recycling endosomes. However, there are data supporting the idea that Rab7 late endosomes can be exocytosed onto the soma membrane. In a carcinoma cell line, $a 5 \beta 1$ integrin is co-localized with Rab7 and LAMP1, which typically mark late endosomes/lysosomes. Instead of degrading, however, $a 5 \beta 1$ integrin is re-directed from a degradative fate to the plasma membrane. Rab25 targets $a 5 \beta 1$ integrin to the lysosome, where an interaction with CLIC3 re-directs its fate from degradation to recycling on the plasma membrane (Dozynkiewicz et al., 2012). Therefore, it is possible that the retrograde signaling endosome is Rab7containing and then gets deposited on the soma membrane, re-internalized, and sorted into a recycling endosome to maintain its lifetime. Another example of this is the lysosomal secretion pathway that is regulated by Rab27 and Rab3. Rab27 and Rab3 are involved in the maturation and function of secretory lysosomes, which are deposited on the plasma membrane to secrete their contents. Both of these are potentially pathways that TrkA could take to re-direct its fate from degradative to recycling, and reconcile the findings that retrograde TrkA signaling endosomes are present in both Rab7 and Rab11 endosomes in the soma. Regulating the trafficking of TrkA in the soma could thus profoundly affect the duration of its associated signaling.

It is possible that the role of signaling transcytosis is to increase the signaling endosome's lifetime, thus trafficking affects signaling. It is unclear, though, what prompts the signaling endosome to undergo this trafficking step. Does signaling affect trafficking by inducing signaling transcytosis? It is still unclear what initiates signaling transcytosis, and currently only one player has been identified: signaling transcytosis is facilitated by the actin-binding protein Coronin1a (Coro1a, aka Coronin 1). The role of Coronin1a will be discussed in the next section.

Trafficking Affects Signaling: Trk Receptor Signaling to Dendrites-BDNF and TrkB have long been known to be necessary for correct dendrite development in many neurons in the central nervous system (Lu, 2003; Miller and Kaplan, 2003; Kuczewski et al., 2010; Leal et al., 2015). In addition to local signaling, BDNF and TrkB retrograde signaling 
is critical for the correct formation of dendrites (Zhou et al., 2012). TrkB is transported retrogradely by attaching to dynein through the adaptor protein snapin. When snapin is lost in the knockout animal, TrkB is not adequately transported retrogradely from the axon, and when BDNF is added in the distal axon of compartmentalized cultures it is insufficient to phosphorylate CREB in the cell body. Additionally, knockout of snapin reduces dendrite arborization, however whether or not this is a result of retrogradely transported TrkB signaling endosomes was not directly tested (Zhou et al., 2012). TrkA retrograde signaling has also been linked to deficits in synapse development. Interestingly, a subset of retrogradely derived TrkA signaling endosomes are transported into dendrites to support synapse development (Sharma et al., 2010).

\section{SAME PROTEIN, DIFFERENT FUNCTION: WHY THE LOCATION OF THE SIGNALING ENDOSOME DETERMINES THE FUNCTIONAL OUTCOME}

Because neurons are such large and polarized cells, it is not surprising that they have adapted unique trafficking mechanisms. Among these strategies are the expression of neuron-specific trafficking proteins and the neuronal-adapted use of ubiquitously expressed trafficking proteins (Yap and Winckler, 2012). TrkA function is critical for multiple aspects of peripheral neurons' survival and development, and it is therefore not surprising that its effectors play multiple roles. Two effectors for TrkA, Calcineurin and Coronin1a, have both been shown to play multiple and sometimes opposing roles with respect to TrkA trafficking.

\section{Calcineurin: Increased Axon Branching Through Local and Transcriptional Mechanisms}

Local TrkA signaling in the distal axon is responsible for axonal extension and innervation. Upon endocytosis in the distal axon, TrkA signals locally to promote branching and innervation of the target tissue. In order for this to occur, TrkA must be correctly endocytosed into a SE with NGF. NGF itself is integral in the internalization of TrkA through induction of the PLC $\gamma$ pathway, one of the major pathways evoked by TrkA. NGF causes the phosphorylation of TrkA on many sites, including one that allows it to recruit and activate PLC $\gamma$ (Harrington et al., 2011).

Signaling downstream of NGF-TrkA is required for its own endocytosis. PLC $\gamma$ activation leads to an increase in intracellular calcium resulting in activation of calcineurin, a serine/ threonine phosphatase activated by calcium. Activated calcineurin can act on a variety of proteins, including the GTPase dynamin. Dynamin promotes endocytosis by pinching off an endosome from the plasma membrane, allowing internalization of the endosome. Interestingly, dynamin function is critical to NGF/TrkA signaling (Riccio et al., 1997; Zhang et al., 2000; Watson et al., 2001; Ye et al., 2003). NGF treatment in the distal axons of SCG neurons causes PLC $\gamma$ activation, which activates calcineurin, which then dephosphorylates and activates dynamin. Activation of dynamin causes it to pinch off the NGF-TrkA endosome, and allows internalization of the NGF-TrkA complex. When calcineurin is inhibited in the distal axon, TrkA endocytosis is depressed, resulting in reduced axon outgrowth as well as reduced NGF-TrkA dependent transcription (Bodmer et al., 2011). Kuruvilla et al. elegantly demonstrate a local requirement of calcineurin and dynamin for endosome internalization at distal axons (Bodmer et al., 2011). Importantly, disruption of 
these signaling molecules perturbs trafficking of the signaling endosome as well as the transcriptional programs induced once NGF-TrkA arrives back to the cell body. As such it is difficult to distinguish whether the impaired axon growth phenotypes that the authors observed are due purely to impaired local signaling or to a lack of NGF dependent transcription.

Intriguingly, the signaling that is required for signaling endosome internalization and trafficking appears to be specific to NGF. During development, SCG neurons extend their axons along blood vessels until they reach their final target and encounter NGF. Blood vessels express the low-affinity ligand for TrkA, NT-3. The NT-3-TrkA complex does not induce the same signaling cascades as the NGF-TrkA complex. Indeed, NT-3 binding to TrkA does not result in activation of PLC $\gamma$ or calcineurin (Bodmer et al., 2011). It has been suggested that, because NT3-TrkA does not signal retrogradely, the majority of its influence on axon outgrowth is local (Kuruvilla et al., 2004). In agreement, the neuron-specific calcineurin KO mouse shows correct axonal extension along blood vessels, where NT-3 binds to TrkA, but an inability to fully innervate the target tissue, where the axon changes preference from NT3 to NGF. The importance of this relationship is underscored by its role in Down's Syndrome. In Down's Syndrome, the calcineurin inhibitor RCAN1 is overexpressed due to gene duplication. This results in depression of calcineurin signaling, leading to inhibition of dynamin1 internalization of TrkA and subsequent cell death (Patel et al., 2015). The relationship between NGF-TrkA, PLC-gamma, and calcineurin at the distal axon is an example of how these signaling proteins interact at a particular locale to promote a function, in this case signaling endosome internalization. However these players also interact in another locale, the cell body, to endow the signaling endosome with a distinct set of abilities such as evading lysosomal fusion and promoting transcription.

Upon arrival to the soma, signaling endosome dependent activation of calcineurin promotes evasion of lysosomal fusion as well as dephosphorylation of the transcription factor NFAT (Graef et al., 2003; Bodmer et al., 2011; Suo et al., 2014). Dephosphorylation of NFAT exposes its nuclear localization signal and allows it to enter the nucleus (Graef et al., 2003). NFAT KO mice are phenotypically similar to calcineurin KO, in that they have dampened innervation of the target tissue. This indicates that calcineurin is able to promote axon growth both through local signaling and through the induction of NFAT transcriptional signaling (Graef et al., 2003; Bodmer et al., 2011). The question remains, how the axon growth program changes as a function of engagement of the NGF-TrkA dependent transcriptional program. This idea will be discussed further in the next section. This clearly demonstrates two distinct roles for calcineurin in promoting axon outgrowth: local signaling in the distal axon via dynamin dephosphorylation, and activation of the transcription factor NFAT4 in the soma. These kinds of bifurcations in the downstream effectors thus allow for diverse and location-specific signaling downstream of NGF-TrkA.

\section{Coronin1a Decreases Axon Branching and Is Necessary for Lysosomal Evasion}

Coronin1a is a well described actin binding protein. In addition, Coronin1a was shown to associate with endosomes in macrophages, where it acts to shield pathogenic mycobacteria from lysosomal fusion. Interestingly, protection from lysosomal fusion via Coronin1A 
occurs through mediation of calcium release and induction of calcineurin activity (Jayachandran et al., 2007; Jayachandran and Pieters, 2015). Coronin1a plays a similar role for the signaling endosome in sympathetic neurons. The loss of Coronin1a in Corola KO mice results in decreased survival of SCGs due to a decrease in NGF-TrkA survival signaling. Surprisingly, loss of Coronin1A causes a dramatic reduction of signaling transcytosis in sympathetic neurons. Additionally, Corola KO sympathetic neurons showed accelerated fusion of TrkA signaling endosomes with lysosomes (Suo et al., 2014). However, the exact mechanism by which Coronin1 a protects the signaling endosome is not clear. It is likely that, similar to its role in mycobacteria, Coronin1a induces calcium release and calcineurin activity, and this signaling is sufficient to evade lysosomal fusion (Jayachandran et al., 2007). Indeed, Coronin1 a has been shown to induce these same signaling cascades in sympathetic neurons (Suo et al., 2014). Interestingly, the premature fusion of SEs with lysosomes can be mimicked in wild-type neurons when the cell bodies are treated with a calcineurin inhibitor, potentially pointing toward a role for Coronin1a in the soma in relation to calcium regulation (Suo et al., 2014). At this time Coronin1a is the only known effector of signaling transcytosis, and because loss of lysosomal avoidance and loss of signaling transcytosis both occur in the Coronin1A KO (Suo et al., 2014), it is currently impossible to uncouple signaling from trafficking and determine the sequence and importance of both events.

While correct innervation of the target tissue is a necessity, axonal overgrowth can also be a problem. As previously mentioned, Coronin1a is an actin-binding protein that associates with the signaling endosome in the soma. Additionally, Coronin1a plays a role in the distal axon where it regulates axonal growth and branching. Without Coronin1a, sympathetic axons overgrow and overbranch. This overgrowth is due to dysregulated PLC $\gamma$ signaling and loss of calcium release (Suo et al., 2015). It is unclear whether or not TrkA must be internalized in order for this signaling to take place and whether or not the actin binding properties of Coronin1a are responsible for this phenotype. Similarly to calcineurin, Coronin1a's role in the distal axon is distinctly different from that in the soma, where it acts to shield the SE from lysosomal fusion (Suo et al., 2014). This stark contrast between seemingly promoting TrkA survival in the soma and preventing overgrowth in the distal axon highlights the different roles that the same effector can play based on its location.

Intriguingly, Coronin1 a has also been shown to play a role in endocytosis in macrophages (BoseDasgupta and Pieters, 2014). Upon mycobacterium infection, a macrophage switches its primary mode of endocytosis from phagocytosis to macropinocytosis, which aids the macrophage in clearance of the bacteria as mycobacteria are efficient at evading degradation in a phagosome. Coronin1 a has been shown to play an important role in this switch as expression of a phosphomimetic Coronin1a isoform is able to initiate PI3K signaling and macropinocytosis (BoseDasgupta and Pieters, 2014). In sympathetic neurons, NGF signaling leads to an increase in the expression of Pincher, which directs TrkA/NGF complexes to be internalized through macropinocytosis (Shao et al., 2002; Valdez et al., 2005; Philippidou et al., 2011). However, there is also ample evidence that clathrin-mediated endocytosis is responsible for endocytosis of the signaling endosome (Grimes et al., 1996; Beattie et al., 2000; Howe et al., 2001; De Pablo et al., 2008; Zheng et al., 2008). While a role for Coronin1a in switching internalization mechanisms of TrkA from clathrin-mediated 
endocytosis to Pincher-mediated macropinocytosis has not been shown, it is interesting to speculate about the potential role of this novel effector.

\section{CONCLUSION}

Trk receptors are complex in terms of their signaling and trafficking capacities. They are able to influence a cell's survival, axon extension, dendrite formation, and synapse maintenance through complex connections and feedback loops between signaling and trafficking. Disruption of neurotrophin-dependent processes have been long attributed to signaling deficits, however the field now recognizes the importance of trafficking in shaping Trk receptors' signaling capabilities and vice versa. There are many remaining questions about Trk receptor trafficking including switching from constitutive endocytosis to anterograde transport, the type of endosome that carries Trk back from the axon, how the signaling endosome is able to evade lysosomal fusion for an elongated period of time, and how it is able to be transported into the dendrites. These are questions common to many other neuronal RTKs, even though each receptor's action and transport may be different. These are questions common to many neuronal RTKs in addition to Trk receptors, even though each receptor may act and transport in different ways. Therefore, understanding the specifics of Trk receptor trafficking will yield important insights into the receptor tyrosine kinase trafficking field as a whole.

\section{Acknowledgments}

Contract grant sponsors: NIH-NINDS-R01NS072388 (to C.D.D.), NINDS-R01NS083378 (to B.W.), and a Wagner Fellowship (to K.B.).

The authors thank Dr. Chan Choo Yap for critical reading of the manuscript.

\section{References}

Arantes RME, Andrews NW. A role for synaptotag-min VII-regulated exocytosis of lysosomes in neurite outgrowth from primary sympathetic neurons. J Neurosci. 2006; 26:4630-4637. [PubMed: $16641243]$

Arimura N, Kimura T, Nakamuta S, Taya S, Funahashi Y, Hattori A, Shimada A, Ménager C, Kawabata S, Fujii K, et al. Anterograde transport of TrkB in axons is mediated by direct interaction with Slp1 and Rab27. Dev Cell. 2009; 16:675-686. [PubMed: 19460344]

Ascaño M, Richmond A, Borden P, Kuruvilla R. Axonal targeting of Trk receptors via transcytosis regulates sensitivity to neurotrophin responses. J Neurosci. 2009; 29:11674-11685. [PubMed: 19759314]

Barker PA. p75NTR: A study in contrasts. Cell Death Differ. 1998; 5:346-356. [PubMed: 10200483]

Barr F, Lambright DG. Rab GEFs and GAPs. Curr Opin Cell Biol. 2010; 22:461-470. [PubMed: 20466531]

Beattie EC, Howe CL, Wilde A, Brodsky FM, Mobley WC. NGF Signals through TrkA to increase clathrin at the plasma membrane and enhance clathrin-mediated membrane trafficking. J Neurosci. 2000; 20:7325-7333. [PubMed: 11007890]

Blott EJ, Griffiths GM. Secretory lysosomes. Nat Rev Mol Cell Biol. 2002; 3:122-131. [PubMed: 11836514]

Bodmer D, Ascaño M, Kuruvilla R. Isoform-specific dephosphorylation of dynamin1 by calcineurin couples neurotrophin receptor endocytosis to axonal growth. Neuron. 2011; 70:1085-1099. [PubMed: 21689596] 
Bonifacino JS. Adaptor proteins involved in polarized sorting. J Cell Biol. 2014; 204:7-17. [PubMed: 24395635]

BoseDasgupta S, Pieters J. Inflammatory stimuli reprogram macrophage phagocytosis to macropinocytosis for the rapid elimination of pathogens. PLoS Pathog. 2014; 10

Bowman AB, Kamal A, Ritchings BW, Philp AV, McGrail M, Gindhart JG, Goldstein LS. Kinesindependent axonal transport is mediated by the sunday driver (SYD) protein. Cell. 2000; 103:583594. [PubMed: 11106729]

Bucci C, Alifano P, Cogli L. The role of rab proteins in neuronal cells and in the trafficking of neurotrophin receptors. Membranes (Basel). 2014; 4:642-677. [PubMed: 25295627]

Bueker ED. Implantation of tumors in the hind limb field of the embryonic chick and the developmental response of the lumbosacral nervous system. Anat Rec. 1948; 102:369-389. [PubMed: 18098427]

Burd C, Cullen PJ. Retromer: A master conductor of endosome sorting. Cold Spring Harb Perspect Biol. 2014; 6:a016774. [PubMed: 24492709]

Callaghan J, Nixon S, Bucci C, Toh BH, Stenmark H. Direct interaction of EEA1 with Rab5b. Eur J Biochem. 1999; 265:361-366. [PubMed: 10491193]

Campenot RB. Local control of neurite development by nerve growth factor. Proc Natl Acad Sci U S A. 1977; 74:4516-4519. [PubMed: 270699]

Chiang L, Ngo J, Schechter JE, Karvar S, Tolmachova T, Seabra MC, Hume AN, Hamm-Alvarez SF. $\mathrm{Rab} 27 \mathrm{~b}$ regulates exocytosis of secretory vesicles in acinar epithelial cells from the lacrimal gland. Am J Physiol Cell Physiol. 2011; 301:C507-C521. [PubMed: 21525430]

Crowley C, Spencer SD, Nishimura MC, Chen KS, Pitts-Meek S, Armanini MP, Ling LH, McMahon SB, Shelton DL, Levinson AD, et al. Mice lacking nerve growth factor display perinatal loss of sensory and sympathetic neurons yet develop basal forebrain cholinergic neurons. Cell. 1994; 76:1001-1011. [PubMed: 8137419]

Deinhardt K, Salinas S, Verastegui C, Watson R, Worth D, Hanrahan S, Bucci C, Schiavo G. Rab5 and Rab7 control endocytic sorting along the axonal retrograde transport pathway. Neuron. 2006; 52:293-305. [PubMed: 17046692]

Delcroix JD, Valletta JS, Wu C, Hunt SJ, Kowal AS, Mobley WC. NGF signaling in sensory neurons: Evidence that early endosomes carry NGF retrograde signals. Neuron. 2003; 39:69-84. [PubMed: 12848933]

Deppmann CD, Mihalas S, Sharma N, Lonze BE, Niebur E, Ginty DD. A model for neuronal competition during development. Science. 2008; 320:369-373. [PubMed: 18323418]

Deshmukh M, Vasilakos J, Deckwerth TL, Lampe PA, Shivers BD, Johnson EM. Genetic and metabolic status of NGF-deprived sympathetic neurons saved by an inhibitor of ICE family proteases. J Cell Biol. 1996; 135:1341-1354. [PubMed: 8947555]

Detwiler SR. Experiments on the transplantation of limb in amblystoma. The innervation and function of limbs transplanted after the outgrowth of peripheral nerves. Am J Anat. 1924; 33:407-419.

DiStefano PS, Friedman B, Radziejewski C, Alexander C, Boland P, Schick CM, Lindsay RM, Wiegand SJ. The neurotrophins BDNF, NT-3, and NGF display distinct patterns of retrograde axonal transport in peripheral and central neurons. Neuron. 1992; 8:983-993. [PubMed: 1375039]

Dozynkiewicz, Ma, Jamieson, NB., Macpherson, I., Grindlay, J., van den Berghe, PVE., von Thun, A., Morton, JP., Gourley, C., Timpson, P., Nixon, C., et al. Rab25 and CLIC3 collaborate to promote integrin recycling from late endosomes/lysosomes and drive cancer progression. Dev Cell. 2012; 22:131-145. [PubMed: 22197222]

Esteves da Silva M, Adrian M, Schatzle P, Lipka J, Watanabe T, Cho S, Futai K, Wierenga CJ, Kapitein LC, Hoogenraad CC. Positioning of AMPA receptor-containing endosomes regulates synapse architecture. Cell Rep. 2015:933-943. [PubMed: 26565907]

Eva R, Dassie E, Caswell PT, Dick G, Ffrench-Constant C, Norman JC, Fawcett JW. Rab11 and its effector Rab coupling protein contribute to the trafficking of beta 1 integrins during axon growth in adult dorsal root ganglion neurons and PC12 cells. J Neurosci. 2010; 30:11654-11669. [PubMed: 20810886]

Glebova NO, Ginty DD. Growth and survival signals controlling sympathetic nervous system development. Annu Rev Neurosci. 2005; 28:191-222. [PubMed: 16022594] 
Graef IA, Wang F, Charron F, Chen L, Neilson J, Tessier-Lavigne M, Crabtree GR. Neurotrophins and netrins require calcineurin/NFAT signaling to stimulate outgrowth of embryonic axons. Cell. 2003; 113:657-670. [PubMed: 12787506]

Grimes ML, Zhou J, Beattie EC, Yuen EC, Hall DE, Valletta JS, Topp KS, LaVail JH, Bunnett NW, Mobley WC. Endocytosis of activated TrkA: Evidence that nerve growth factor induces formation of signaling endosomes. J Neurosci. 1996; 16:7950-7964. [PubMed: 8987823]

Grosshans BL, Ortiz D, Novick P. Rabs and their effectors: Achieving specificity in membrane traffic. Proc Natl Acad Sci U S A. 2006; 103:11821-11827. [PubMed: 16882731]

Hamburger V. The effects of wing bud extirpation on the development of the central nervous system in chick embryos. J Exp Zool. 1934; 68:449-494.

Hanson PI, Cashikar A. Multivesicular Body Morphogenesis. Annu Rev Cell Dev Biol. 2012; 28:337362. [PubMed: 22831642]

Harrington AW, St Hillaire C, Zweifel LS, Glebova NO, Philippidou P, Halegoua S, Ginty DD. Recruitment of actin modifiers to TrkA endosomes governs retrograde NGF signaling and survival. Cell. 2011; 146:421-434. [PubMed: 21816277]

Heerssen HM, Pazyra MF, Segal Ra. Dynein motors transport activated Trks to promote survival of target-dependent neurons. Nat Neurosci. 2004; 7:596-604. [PubMed: 15122257]

Henne WM, Stenmark H, Emr SD. Molecular mechanisms of the membrane sculpting ESCRT pathway. Cold Spring Harb Perspect Biol. 2013; 5:a016766. [PubMed: 24003212]

Horgan CP, Hanscom SR, Jolly RS, Futter CE, McCaffrey MW. Rab11-FIP3 links the Rab11 GTPase and cytoplasmic dynein to mediate transport to the endosomal-recycling compartment. J Cell Sci. 2010; 123:181-191. [PubMed: 20026645]

Howe CL, Mobley WC. Signaling endosome hypothesis: a cellular mechanism for long distance communication. J Neurobiol. 2004; 58:207-216. [PubMed: 14704953]

Howe CL, Valletta JS, Rusnak AS, Mobley WC. NGF signaling from clathrin-coated vesicles: Evidence that signaling endosomes serve as a platform for the Ras-MAPK pathway. Neuron. 2001; 32:801-814. [PubMed: 11738027]

Huang EJ, Reichardt LF. Trk receptors: Roles in neuronal signal transduction. Annu Rev Biochem. 2003; 72:609-642. [PubMed: 12676795]

Huang SH, Duan S, Sun T, Wang J, Zhao L, Geng Z, Yan J, Sun HJ, Chen ZY. JIP3 mediates TrkB axonal anterograde transport and enhances BDNF signaling by directly bridging TrkB with kinesin-1. J Neurosci. 2011; 31:10602-10614. [PubMed: 21775604]

Huotari J, Helenius A. Endosome maturation. Embo J. 2011; 30:3481-3500. [PubMed: 21878991]

Hutagalung AH, Novick PJ. Role of Rab GTPases in membrane traffic and cell physiology. Physiol Rev. 2011; 91:119-149. [PubMed: 21248164]

Ikonen E, Parton RG, Hunziker W, Simons K, Dotti CG. Transcytosis of the polymeric immunoglobulin receptor in cultured hippocampal neurons. Curr Biol. 1993; 3:635-644. [PubMed: 15335854]

Jayachandran R, Pieters J. Regulation of immune cell homeostasis and function by coronin 1. Int Immunopharmacol. 2015; 28:825-828. [PubMed: 25882105]

Jayachandran R, Sundaramurthy V, Combaluzier B, Mueller P, Korf H, Huygen K, Miyazaki T, Albrecht I, Massner J, Pieters J. Survival of mycobacteria in macrophages is mediated by coronin 1-dependent activation of calcineurin. Cell. 2007; 130:37-50. [PubMed: 17632055]

Jovic M, Sharma M, Rahajeng J, Caplan S. The early endosome: A busy sorting station for proteins at the crossroads. Histol Histopathol. 2010; 25:99-112. [PubMed: 19924646]

Kaplan DR, Stephens RM. Neurotrophin signal transduction by the Trk receptor. J Neurobiol. 1994; 25:1404-1417. [PubMed: 7852994]

Klumperman J, Raposo G. The Complex Ultrastructure of the Endolysosomal System. Cold Spring Harb Perspect Biol. 2014; 6:a016857-a016857. [PubMed: 24851870]

Kuczewski N, Porcher C, Gaiarsa JL. Activity-dependent dendritic secretion of brain-derived neurotrophic factor modulates synaptic plasticity. Eur J Neurosci. 2010; 32:1239-1244. [PubMed: 20880359] 
Kuruvilla R, Zweifel LS, Glebova NO, Lonze BE, Valdez G, Ye H, Ginty DD. A neurotrophin signaling cascade coordinates sympathetic neuron development through differential control of TrkA trafficking and retrograde signaling. Cell. 2004; 118:243-55. [PubMed: 15260993]

Lasiecka ZM, Yap CC, Katz J, Winckler B. Maturational conversion of dendritic early endosomes and their roles in L1-mediated axon growth. J Neurosci. 2014; 34:14633-14643. [PubMed: 25355216]

Leal G, Afonso PM, Salazar IL, Duarte CB. Regulation of hippocampal synaptic plasticity by BDNF. Brain Res. 2015; 1621:82-101. [PubMed: 25451089]

Lettau M, Kabelitz D, Janssen O. Lysosome-related effector vesicles in T lymphocytes and NK cells. Scand J Immunol. 2015; 82:235-243. [PubMed: 26118957]

Levi-Montalcini R, Hamburger V. Selective growth stimulating effects of mouse sarcoma on the sensory and sympathetic nervous system of the chick embryo. J Exp Zool. 1951; 116:321-361. [PubMed: 14824426]

Lin DC, Quevedo C, Brewer NE, Bell A, Testa JR, Grimes ML, Miller FD, Kaplan DR. APPL1 associates with TrkA and GIPC1 and is required for nerve growth factor-mediated signal transduction. Mol Cell Biol. 2006; 26:8928-8941. [PubMed: 17000777]

Lin X, Yang T, Wang S, Wang Z, Yun Y, Sun L, Zhou Y, Xu X, Akazawa C, Hong W, et al. RILP interacts with HOPS complex via VPS41 subunit to regulate endocytic trafficking. Sci Rep. 2013; 4:7282.

Liu J, Lamb D, Chou MM, Liu YJ, Li G. Nerve growth factor-mediated neurite outgrowth via regulation of Rab5. Mol Biol Cell. 2007; 18:1375-1384. [PubMed: 17267689]

Lu B. BDNF and Activity-Dependent Synaptic Modulation. Learn Mem. 2003; 10:86-98. [PubMed: 12663747]

Marchesin V, Castro-Castro A, Lodillinsky C, Castagnino A, Cyrta J, Bonsang-Kitzis H, Fuhrmann L, Irondelle M, Infante E, Montagnac G, et al. ARF6-JIP3/4 regulate endosomal tubules for MT1MMP exocytosis in cancer invasion. J Cell Biol. 2015; 211:339-358. [PubMed: 26504170]

Meeker RB, Williams KS. The p75 neurotrophin receptor: At the crossroad of neural repair and death. Neural Regen Res. 2015; 10:721-725. [PubMed: 26109945]

Miller FD, Kaplan DR. Signaling mechanisms underlying dendrite formation. Curr Opin Neurobiol. 2003; 13:391-398. [PubMed: 12850225]

Mitchell DJ, Blasier KR, Jeffery ED, Ross MW, Pullikuth AK, Suo D, Park J, Smiley WR, Lo KWH, Shabanowitz J, et al. Trk activation of the ERK1/2 kinase pathway stimulates intermediate chain phosphorylation and recruits cytoplasmic dynein to signaling endosomes for retrograde axonal transport. J Neurosci. 2012; 32:15495-15510. [PubMed: 23115187]

Naslavsky N, Caplan S. EHD proteins: Key conductors of endocytic transport. Trends Cell Biol. 2011; 21:122-131. [PubMed: 21067929]

De Pablo Y, Pérez-García MJ, Georgieva MV, Sanchis D, Lindqvist N, Soler RM, Comella JX, Llovera $\mathrm{M}$. Tyr-701 is a new regulatory site for neurotrophin receptor TrkA trafficking and function. $\mathrm{J}$ Neurochem. 2008; 104:124-139. [PubMed: 18173729]

Patapoutian A, Reichardt LF. Trk receptors: Mediators of neurotrophin action. Curr Opin Neurobiol. 2001; 11:272-280. [PubMed: 11399424]

Patel A, Yamashita N, Ascaño M, Bodmer D, Boehm E, Bodkin-Clarke C, Ryu YK, Kuruvilla R. RCAN1 links impaired neurotrophin trafficking to aberrant development of the sympathetic nervous system in Down syndrome. Nat Commun. 2015; 6:10119. [PubMed: 26658127]

Philippidou P, Valdez G, Akmentin W, Bowers WJ, Federoff HJ, Halegoua S. Trk retrograde signaling requires persistent, Pincher-directed endosomes. Proc Natl Acad Sci U S A. 2011; 108:852-857. [PubMed: 21187387]

Riccio A, Pierchala BA, Ciarallo CL, Ginty DD. An NGF-TrkA-mediated retrograde signal to transcription factor CREB in sympathetic neurons. Science. 1997; 277:1097-1100. [PubMed: 9262478]

Riccio A, Ahn S, Davenport CM, Blendy JA, Ginty DD. Mediation by a CREB family transcription factor of NGF-dependent survival of sympathetic neurons. Science. 1999; 286:2358-2361. [PubMed: 10600750]

Saragovi HU, Zheng W, Maliartchouk S, DiGugliemo GM, Mawal YR, Kamen A, Woo SB, Cuello aC, Debeir T, Neet KE. A TrkA-selective, fast internalizing nerve growth factor-antibody complex 
induces trophic but not neuritogenic signals. J Biol Chem. 1998; 273:34933-34940. [PubMed: 9857023]

Saxena S, Bucci C, Weis J, Kruttgen A. The small GTPase Rab7 controls the endosomal trafficking and neuritogenic signaling of the nerve growth factor receptor TrkA. J Neurosci. 2005; 25:1093010940. [PubMed: 16306406]

Schafer JC, Baetz NW, Lapierre LA, McRae RE, Roland JT, Goldenring JR. Rab11-FIP2 interaction with MYO5B regulates movement of Rab11a-containing recycling vesicles. Traffic. 2014; 15:292308. [PubMed: 24372966]

Schonteich E, Wilson GM, Burden J, Hopkins CR, Anderson K, Goldenring JR, Prekeris R. The Rip11/Rab11-FIP5 and kinesin II complex regulates endocytic protein recycling. J Cell Sci. 2008; 121:3824-3833. [PubMed: 18957512]

Scott CC, Vacca F, Gruenberg J. Endosome maturation, transport and functions. Semin Cell Dev Biol. 2014; 31:2-10. [PubMed: 24709024]

Setou M, Seog DH, Tanaka Y, Kanai Y, Takei Y, Kawagishi M, Hirokawa N. Glutamate-receptorinteracting protein GRIP1 directly steers kinesin to dendrites. Nature. 2002; 417:83-87. [PubMed: 11986669]

Shao Y, Akmentin W, Toledo-Aral JJ, Rosenbaum J, Valdez G, Cabot JB, Hilbush BS, Halegoua S. Pincher, a pinocytic chaperone for nerve growth factor/TrkA signaling endosomes. J Cell Biol. 2002; 157:679-691. [PubMed: 12011113]

Sharma N, Deppmann CD, Harrington AW, St Hillaire C, Chen ZY, Lee FS, Ginty DD. Long-distance control of synapse assembly by target-derived NGF. Neuron. 2010; 67:422-434. [PubMed: 20696380]

Sheff DR, Daro EA, Hull M, Mellman I. The receptor recycling pathway contains two distinct populations of early endosomes with different sorting functions. J Cell Biol. 1999; 145:123-139. [PubMed: 10189373]

Simon GC, Prekeris R. Mechanisms regulating targeting of recycling endosomes to the cleavage furrow during cytokinesis. Biochem Soc Trans. 2008; 36:391-394. [PubMed: 18481966]

Simonsen A, Lippé R, Christoforidis S, Gaullier JM, Brech A, Callaghan J, Toh BH, Murphy C, Zerial M, Stenmark H. EEA1 links PI(3)K function to Rab5 regulation of endosome fusion. Nature. 1998; 394:494-498. [PubMed: 9697774]

Smeyne RJ, Klein R, Schnapp A, Long LK, Bryant S, Lewin A, Lira SA, Barbacid M. Severe sensory and sympathetic neuropathies in mice carrying a disrupted Trk/NGF receptor gene. Nature. 1994; 368:246-249. [PubMed: 8145823]

Snider WD. Functions of the neurotrophins during nervous system development: What the knockouts are teaching us. Cell. 1994; 77:627-638. [PubMed: 8205613]

Suo D, Park J, Harrington AW, Zweifel LS, Mihalas S, Deppmann CD. Coronin-1 is a neurotrophin endosomal effector that is required for developmental competition for survival. Nat Neurosci. 2014; 17:36-45. [PubMed: 24270184]

Suo D, Park J, Young S, Makita T, Deppmann CD. Coronin-1 and Calcium Signaling Governs Sympathetic Final Target Innervation. J Neurosci. 2015; 35:3893-3902. [PubMed: 25740518]

Takano T, Tomomura M, Yoshioka N, Tsutsumi K, Terasawa Y, Saito T, Kawano H, Kamiguchi H, Fukuda M, Hisanaga S. LMTK1/AATYK1 is a novel regulator of axonal outgrowth that acts via Rab11 in a Cdk5-dependent manner. J Neurosci. 2012; 32:6587-6599. [PubMed: 22573681]

Vaegter CB, Jansen P, Fjorback AW, Glerup S, Skeldal S, Kjolby M, Richner M, Erdmann B, Nyengaard JR, Tessarollo L, et al. Sortilin associates with Trk receptors to enhance anterograde transport and neurotrophin signaling. Nat Neurosci. 2011; 14:54-61. [PubMed: 21102451]

Valdez G, Akmentin W, Philippidou P, Kuruvilla R, Ginty DD, Halegoua S. Pincher-mediated macroendocytosis underlies retrograde signaling by neurotrophin receptors. J Neurosci. 2005; 25:5236-5247. [PubMed: 15917464]

Wandinger-Ness A, Zerial M. Rab Proteins and the Compartmentalization of the Endosomal System. Cold Spring Harb Perspect Biol. 2014; 6:a022616. [PubMed: 25341920]

Wang T, Hong W. RILP interacts with VPS22 and VPS36 of ESCRT-II and regulates their membrane recruitment. Biochem Biophys Res Commun. 2006; 350:413-423. [PubMed: 17010938] 
Watson FL, Heerssen HM, Bhattacharyya a, Klesse L, Lin MZ, Segal Ra. Neurotrophins use the Erk5 pathway to mediate a retrograde survival response. Nat Neurosci. 2001; 4:981-988. [PubMed: 11544482]

Wilson JM, de Hoop M, Zorzi N, Toh BH, Dotti CG, Parton RG. EEA1, a tethering protein of the early sorting endosome, shows a polarized distribution in hippocampal neurons, epithelial cells, and fibroblasts. Mol Biol Cell. 2000; 11:2657-2671. [PubMed: 10930461]

Wisco D. Uncovering multiple axonal targeting pathways in hippocampal neurons. J Cell Biol. 2003; 162:1317-1328. [PubMed: 14517209]

Wojnacki J, Galli T. Membrane traffic during axon development. Dev Neurobiol. 2016; 76:1185-1200. [PubMed: 26945675]

Woodman PG, Futter CE. Multivesicular bodies: Coordinated progression to maturity. Curr Opin Cell Biol. 2008; 20:408-414. [PubMed: 18502633]

Yamashita N, Kuruvilla R. Neurotrophin signaling endosomes: Biogenesis, regulation, and functions. Curr Opin Neurobiol. 2016; 39:139-145. [PubMed: 27327126]

Yamazaki T, Selkoe DJ, Koo EH. Trafficking of cell surface beta-amyloid precursor protein: Retrograde and transcytotic transport in cultured neurons. J Cell Biol. 1995; 129:431-442. [PubMed: 7721945]

Yap CC, Winckler B. Harnessing the power of the endosome to regulate neural development. Neuron. 2012; 74:440-451. [PubMed: 22578496]

Yap CC, Wisco D, Kujala P, Lasiecka ZM, Cannon JT, Chang MC, Hirling H, Klumperman J, Winckler B. The somatodendritic endosomal regulator NEEP21 facilitates axonal targeting of L1/ NgCAM. J Cell Biol. 2008; 180:827-842. [PubMed: 18299352]

Ye H, Kuruvilla R, Zweifel LS, Ginty DD. Evidence in support of signaling endosome-based retrograde survival of sympathetic neurons. Neuron. 2003; 39:57-68. [PubMed: 12848932]

Yun HJ, Kim H, Ga I, Oh H, Ho DH, Kim J, Seo H, Son I, Seol W. An early endosome regulator, Rab5b, is an LRRK2 kinase substrate. J Biochem. 2015; 157:485-495. [PubMed: 25605758]

Zhang K, Fishel Ben Kenan R, Osakada Y, Xu W, Sinit RS, Chen L, Zhao X, Chen JY, Cui B, Wu C. Defective axonal transport of Rab7 GTPase results in dysregulated trophic signaling. J Neurosci. 2013; 33:7451-7462. [PubMed: 23616551]

Zhang Y, Moheban DB, Conway BR, Bhattacharyya a, Segal Ra. Cell surface Trk receptors mediate NGF-induced survival while internalized receptors regulate NGF-induced differentiation. J Neurosci. 2000; 20:5671-5678. [PubMed: 10908605]

Zhen Y, Stenmark H. Cellular functions of Rab GTPases at a glance. J Cell Sci. 2015; 128:3171-3176. [PubMed: 26272922]

Zheng J, Shen WH, Lu TJ, Zhou Y, Chen Q, Wang Z, Xiang T, Zhu YC, Zhang C, Duan S, et al. Clathrin-dependent endocytosis is required for TrkB-dependent Akt-mediated neuronal protection and dendritic growth. J Biol Chem. 2008; 283:13280-13288. [PubMed: 18353779]

Zhou B, Cai Q, Xie Y, Sheng ZH. Snapin recruits dynein to BDNF-TrkB signaling endosomes for retrograde axonal transport and is essential for dendrite growth of cortical neurons. Cell Rep. 2012; 2:42-51. [PubMed: 22840395]

Zweifel LS, Kuruvilla R, Ginty DD. Functions and mechanisms of retrograde neurotrophin signalling. Nat Rev Neurosci. 2005; 6:615-625. [PubMed: 16062170]

Dev Neurobiol. Author manuscript; available in PMC 2017 October 30. 


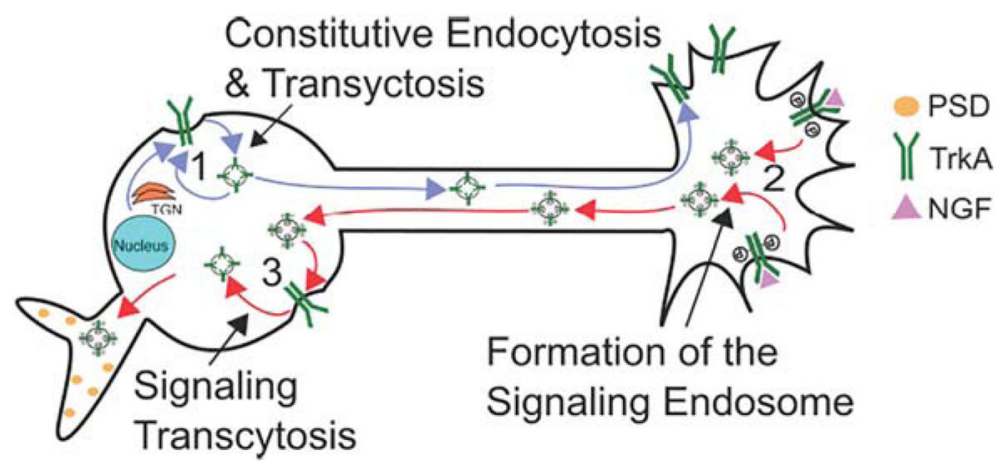

Figure 1.

Internalization and trafficking pathways of TrkA. (1) Constitutive endocytosis and transcytosis to the distal axon before TrkA encounters NGF. (2) TrkA encounters NGF in the distal axon and internalizes into a signaling endosome. The signaling endosomes then traffics retrogradely to the axon. (3) The signaling endosome can then be exocytosed onto the soma membrane and re-internalized during signaling transcytosis. PSD = Postsynaptic Density. [Color figure can be viewed at wileyonlinelibrary.com] 


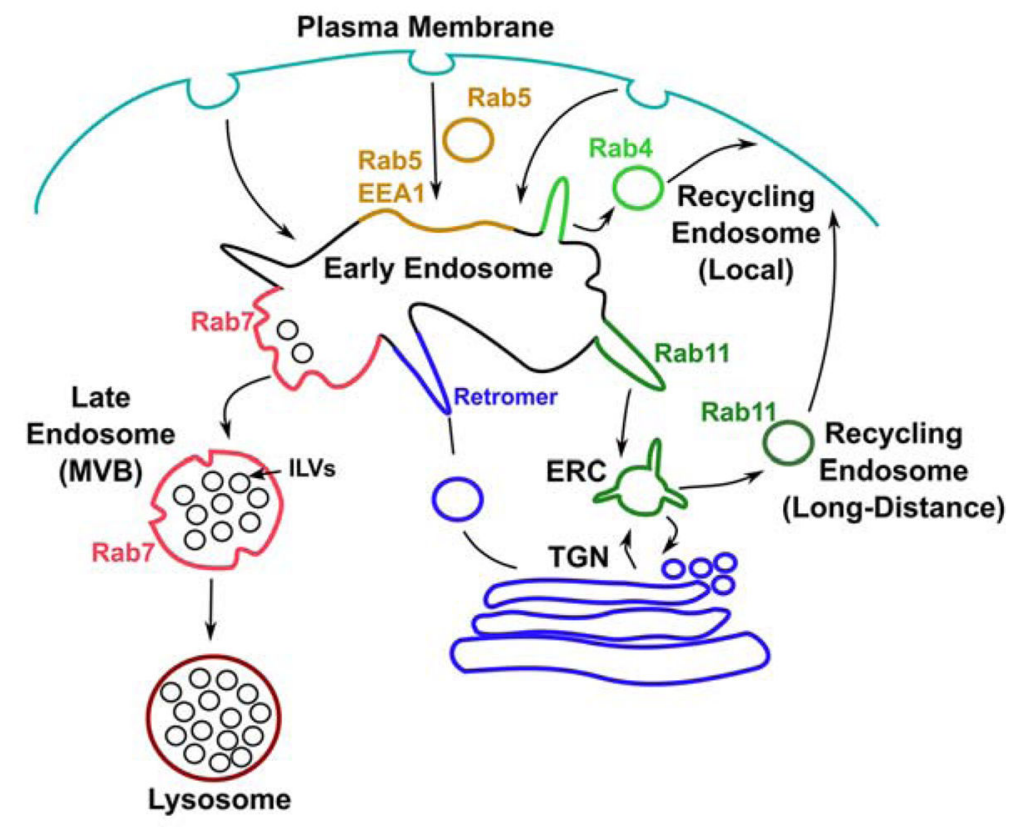

Figure 2.

Basics of endosomal sorting. All internalized cargo gets sorted into the early endosome.

From the early endosome, cargo sorts into distinct compartments which then bud off to form recycling endosomes (Rab4, Rab11), or late endosomes (Rab7) which mature into lysosomes, or sort back to the TGN through retromer. [Color figure can be viewed at wileyonlinelibrary.com] 